\title{
Band alignment between (100)Si and complex rare earth/transition metal oxides
}

\author{
V. V. Afanas'ev a) and A. Stesmans \\ Department of Physics, University of Leuven, Celestijnenlaan 200D, B-3001 Leuven, Belgium \\ C. Zhao and M. Caymax \\ IMEC, Kapeldreef 75, B-3001, Leuven, Belgium
}

T. Heeg and J. Schubert

Institut für Schichten and Grenzfächen, Forschungszentrum Jülich GmbH, D-52425 Jülich, Germany

Y. Jia and D. G. Schlom

Department of Materials Science and Engineering, The Pennsylvania State University, University Park, Pennsylvania 16802

G. Lucovsky

Department of Physics, North Carolina State University, Raleigh, North Carolina 27695-8202

(Received 19 July 2004; accepted 6 October 2004)

\begin{abstract}
The electron energy band alignment between (100)Si and several complex transition/rare earth (RE) metal oxides $\left(\mathrm{LaScO}_{3}, \mathrm{GdScO}_{3}, \mathrm{DyScO}_{3}\right.$, and $\mathrm{LaAlO}_{3}$, all in amorphous form) is determined using a combination of internal photoemission and photoconductivity measurements. The band gap width is nearly the same in all the oxides $(5.6-5.7 \mathrm{eV})$ yielding the conduction and valence band offsets at the $\mathrm{Si}$ /oxide interface of $2.0 \pm 0.1$ and $2.5 \pm 0.1 \mathrm{eV}$, respectively. However, band-tail states are observed and these are associated with Jahn-Teller relaxation of transition metal and RE cations which splits their $d^{*}$ states. (C) 2004 American Institute of Physics. [DOI: 10.1063/1.1829781]
\end{abstract}

Complex rare earth (RE) and transition metal (TM) oxides attract considerable interest as candidate gate insulators for silicon metal-oxide-semiconductor (MOS) devices. Without impairing the high dielectric permittivity, ${ }^{1}$ they allow one, by changing the oxide composition, to engineer the insulator band gap and the band offsets in order to establish energy barriers at the interfaces with $\mathrm{Si}$ sufficiently high to block electron/hole tunneling. ${ }^{2-5}$ While the upper valence band (VB) in the oxides is commonly derived from the same occupied $2 p$ states of $\mathrm{O}$ atoms, the lowest conduction band (CB) consists of empty electron states of metal cations. ${ }^{5}$

There are several factors affecting the electron states in the $\mathrm{CB}$ of a complex oxide as compared to the simple one. At first, in a complex phase the separate TM and RE networks are diluted resulting in a reduced overlap between the states of ions of the same sort. ${ }^{6}$ Second, the reduction in symmetry of the RE/TM ion surrounding, particularly in an amorphous network, may split the unoccupied states. ${ }^{5}$ Finally, the energy position of the unoccupied $4 f$ states in the RE ions is a function of the shell occupancy which causes oxide band gap variation. ${ }^{7}$ To clarify the impact of these factors on the band alignment at the interfaces of $\mathrm{Si}$ with several complex oxides $\left(\mathrm{LaScO}_{3}, \mathrm{GdScO}_{3}, \mathrm{DyScO}_{3}, \mathrm{LaAlO}_{3}\right.$ ) we report here on the direct determination of the oxide band gap width and the $\mathrm{CB} / \mathrm{VB}$ offsets using internal photoemission (IPE) and photoconductivity (PC) measurements.

The samples studied were prepared by deposition of 5 to $39 \mathrm{~nm}$-thick layers of four complex oxides $\left(\mathrm{LaScO}_{3}\right.$, $\left.\mathrm{GdScO}_{3}, \mathrm{DyScO}_{3}, \mathrm{LaAlO}_{3}\right)$ on low-doped $\left(n_{d} \approx 10^{15} \mathrm{~cm}^{-3}\right)$ $n$-type (100)Si substrates covered with a $0.7 \mathrm{~nm}$ chemical $\mathrm{SiO}_{x}$. The deposition was performed at $600{ }^{\circ} \mathrm{C}$ in a 1.2

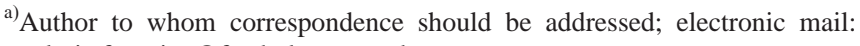
valeri.afanasiev@fys.kuleuven.ac.be $\times 10^{-3}$ mbar $\mathrm{N}_{2}$ ambient using the pulsed laser ablation technique. The film composition was evaluated using Rutherford backscattering spectrometry, giving a RE:TM concentration ratio close to $1: 1$. The $\mathrm{x}$-ray diffraction analysis indicates that all the layers are amorphous and retain this structure even after rapid annealing at temperatures in excess of $800{ }^{\circ} \mathrm{C}$. Some of the samples were thermally oxidized in $\mathrm{O}_{2}$ $(1.1 \mathrm{~atm})$ at $650{ }^{\circ} \mathrm{C}$ for $30 \mathrm{~min}$ to grow a silicate interlayer between $\mathrm{Si}$ and the complex oxide. MOS structures were formed by evaporation of semitransparent ( $15 \mathrm{~nm}$ thick) $\mathrm{Au}$ or $\mathrm{Al}$ electrodes of $0.4 \mathrm{~mm}^{2}$ area. Electrical analysis, in particular, indicates a high dielectric constant of the deposited films $(\kappa \approx 22-24)$. Here we focus on the IPE and PC experiments in the photon energy range $h v=2-6.8 \mathrm{eV}$. The quantum yield $(Y)$ was defined as the photocurrent normalized to the incident photon flux. ${ }^{8}$

IPE/PC spectral curves are shown in Fig. 1(a) as measured in MOS capacitors with Au electrodes. The open and filled symbols correspond to positive and negative bias on the metal, respectively, at an average strength of electric field in the oxide of $1 \mathrm{MV} / \mathrm{cm}$. The field reversal strongly affects the low-photon energy $(2<h v<4.5 \mathrm{eV})$ portion of the spectra suggesting the photocurrent to be due to IPE of charge carriers from the electrodes of the MOS structures into the insulator. In the spectral range $h v>4.5 \mathrm{eV}$ the spectra are similar for both bias polarities indicating intrinsic PC which is also corroborated by the high quantum yield of the process $\left(Y>10^{3}\right)$. To determine the PC threshold (corresponding to the band gap width), the spectral curves are re-plotted in Fig. (b) in $Y^{1 / 2}-h v$ coordinates, as was done before for other amorphous oxide insulators. ${ }^{8,9}$ These plots correspond to the positive metal bias data only-those measured under opposite bias are very similar (not shown). The scandates and $\mathrm{LaAlO}_{3}$ exhibit a well-defined linear portion allowing one to 




FIG. 1. IPE yield (a) and its square root (b) as a function of photon energy measured in $\mathrm{Si} / \mathrm{RETMO}_{3}(\sim 20 \mathrm{~nm}) / \mathrm{Au}$ capacitors. The open and filled symbols correspond to applied oxide electric field of +1 and $-1 \mathrm{MV} / \mathrm{cm}$, respectively. The inset in panel (b) illustrates determination of the IPE thresholds from $Y^{1 / 3}-h \nu$ plot. The IPE and photoconductivity thresholds are indicated by arrows. Lines guide the eye.

infer the oxide band gap value $\left(E_{g}\right)$, as indicated in Fig. 1(b), and a $1-\mathrm{eV}$ wide low-energy "tail" with an apparent threshold $E_{g}^{*} \sim 4.5 \mathrm{eV}$. The PC threshold $E_{g}$ in scandates is found to be in the range of $5.6-5.7 \mathrm{eV}$ and weakly sensitive to the RE type (cf. Table I).

Next we compared the PC spectra in scandates to those measured in $\mathrm{LaAlO}_{3}(\nabla, \boldsymbol{\nabla}$ in Fig. 1). One can see that, despite the narrower band gap of pure $\mathrm{Sc}_{2} \mathrm{O}_{3}\left(E_{g} \approx 4.2 \mathrm{eV}\right.$ as can be evaluated from the optical data in Ref. 10) than that of unannealed deposited $\mathrm{Al}_{2} \mathrm{O}_{3}$ [ $E_{g} \geqslant 6 \mathrm{eV}$ (Refs. 6 and 8)], there is no impact of $\mathrm{Sc}$ substitution by $\mathrm{Al}$ on the $\mathrm{PC}$ spectra. This suggests no measurable contribution of the $\mathrm{Sc} 3 d^{*}$ states [which constitute the lowest $\mathrm{CB}$ in $\mathrm{Sc}_{2} \mathrm{O}_{3}$ (Ref. 11)] to the density of states near the bottom of the scandate CB. Apparently then, the unoccupied states determining the PC originate from the $\mathrm{RE} 5 d^{*}$ states.

In order to determine the band offsets at the interfaces of the complex oxides with $\mathrm{Si}$, we analyzed the low-energy part of the IPE spectra. It appears that the replacement of the Au metal electrode with an $\mathrm{Al}$ one (curves not shown) has no significant effect neither on the spectra measured at

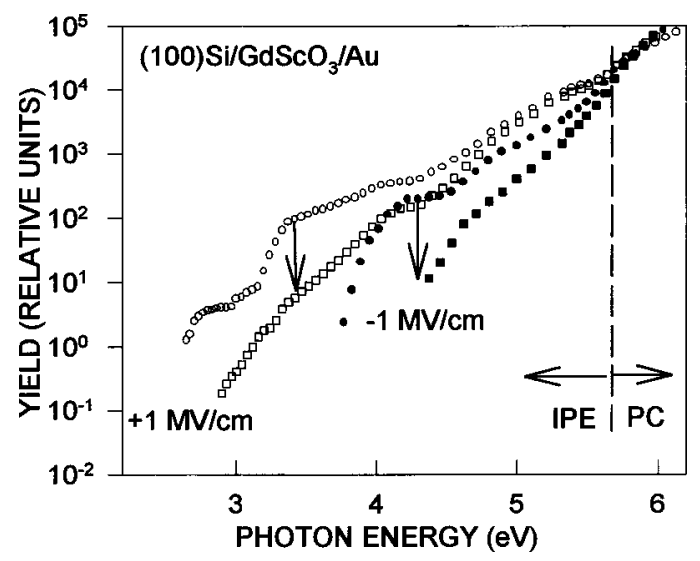

FIG. 2. IPE yield as a function of photon energy measured on (100) $\mathrm{Si} / \mathrm{GdScO}_{3} / \mathrm{Au}$ samples in the as-deposited state (circles) or additionally subjected to $30 \mathrm{~min}$ oxidation in $\mathrm{O}_{2}$ at $650{ }^{\circ} \mathrm{C}$ (squares). The open and filled symbols correspond to an applied oxide electric field bias of +1 and $-1 \mathrm{MV} / \mathrm{cm}$, respectively. Arrows indicate the observed trends.

$+1 \mathrm{MV} / \mathrm{cm}$ field bias nor on those taken with opposite bias. These observations indicate that the photocurrent related to excitation of states close to the Fermi level of these metals [which differ in energy by $\approx 1 \mathrm{eV}$ (Ref. 8)] is negligible as compared to the IPE of electrons and holes from Si into the oxide under either positive or negative bias polarity. This conclusion gained further support from the comparison of the IPE/PC spectra obtained from the MOS structures with as-deposited $\mathrm{GdScO}_{3}$ insulators with those subjected to additional oxidation, as shown in Fig. 2: The PC yield is affected only marginally, while the IPE yield is strongly reduced after additional oxidation indicating suppression of the electron/hole IPE by the grown 2-3-nm-thick $\mathrm{SiO}_{2}$-like interlayer. ${ }^{12}$ The same trend is also observed in the oxidized $\mathrm{LaScO}_{3}, \mathrm{DyScO}_{3}$, and $\mathrm{LaAlO}_{3}$ samples (not shown).

IPE threshold energies were derived using the $Y^{1 / 3}-h v$ plots $^{13}$ as shown in the inset in Fig. 1(b). The electron IPE (open symbols) of both scandates and $\mathrm{LaAlO}_{3}$ exhibits a spectral threshold at $\Phi_{e}=3.0-3.1 \mathrm{eV}$. When extrapolated to zero field bias using the Schottky plot (not shown), this results in an energy barrier between the top of the Si VB and the bottom of the oxide CB of $\Phi_{e}=3.1 \pm 0.1 \mathrm{eV}$. In addition, there is observed a low-energy IPE tail with an effective threshold $\Phi_{e}^{*} \sim 2.2 \mathrm{eV}$ [cf. inset in Fig. 1(b)].

The hole IPE threshold $\Phi_{h}$ was determined in a similar way using the data obtained under negative bias on the metal electrode (filled symbols in Fig. 1). The $Y^{1 / 3}-h v$ plot shown in the inset in Fig. 1(b) indicates that all the complex oxides have the same $\Phi_{h}$ of $3.7 \pm 0.1 \mathrm{eV}$ which, being observed to be nearly independent on electric field strength, represents the barrier between the top of oxide VB and the bottom of $\mathrm{Si}$ CB. This value coincides with $\Phi_{h}$ found at the (100) Si/ $\mathrm{HfO}_{2}$ interface $^{14}$ indicating that the energy position of the $\mathrm{O}$

TABLE I. Energy band diagram parameters of interfaces between Si and different deposited metal oxides.

\begin{tabular}{ccccccc}
\hline \hline Oxide & $\mathrm{LaScO}_{3}$ & $\mathrm{GdScO}_{3}$ & $\mathrm{DyScO}_{3}$ & $\mathrm{LaAlO}_{3}$ & $\mathrm{Al}_{2} \mathrm{O}_{3}$ & $\mathrm{HfO}_{2}$ \\
\hline$E_{g} \pm 0.1 \mathrm{eV}$ & 5.7 & 5.6 & 5.7 & 5.7 & 6.2 & 5.6 \\
$\Phi_{e} \pm 0.1 \mathrm{eV}$ & 3.1 & 3.1 & 3.1 & 3.1 & 3.25 & 3.1 \\
$\Delta E_{c} \pm 0.1 \mathrm{eV}$ & 2.0 & 2.0 & 2.0 & 2.0 & 2.15 & 2.0 \\
$\Phi_{h} \pm 0.1 \mathrm{eV}$ & 3.6 & 3.6 & 3.6 & 3.7 & $\cdots$ & 3.6 \\
$\Delta E_{V} \pm 0.1 \mathrm{eV}$ & 2.5 & 2.5 & 2.5 & 2.6 & 3.0 & 2.5 \\
\hline \hline
\end{tabular}



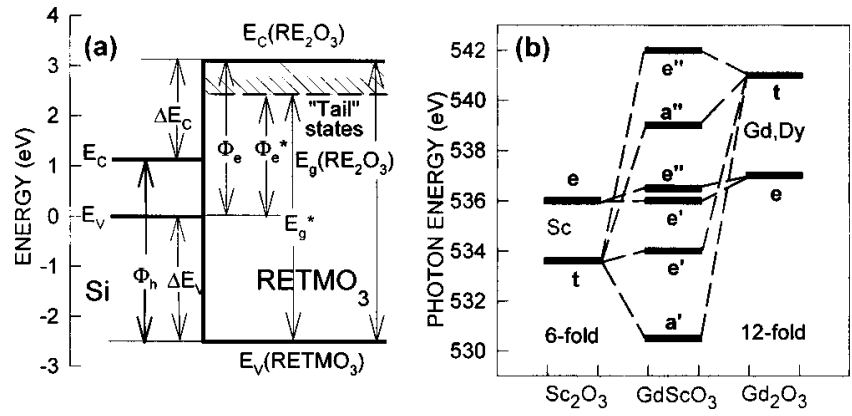

FIG. 3. (a) Energy band diagram of the (100)Si/RETMO ${ }_{3}$ interface inferred from IPE and PC experiments. The origin of the energy scale is placed at the top of $\mathrm{Si} \mathrm{VB}$. The values of the band diagram parameters are listed in Table I for the four oxides studied and for the elemental $\mathrm{Al}_{2} \mathrm{O}_{3}$ and $\mathrm{HfO}_{2}$ (Ref. 8). (b) The scheme of coupling between $d^{*}$ states of Sc and those of Gd, Dy and other $\mathrm{RE} d^{*}$ states in complex oxides, $\mathrm{RE}_{2} \mathrm{O}_{3}$ derived from the $\mathrm{x}$-ray absorption spectra near the $\mathrm{O} K_{1}$ edge.

$2 p$-derived states is nearly unaffected by the composition of the sixth-period cation network. However, the measured oxide band gap and $\Phi_{e}$ values (cf. Table I) suggest a $\approx 0.5 \mathrm{eV}$ downshift of the $\mathrm{Al}_{2} \mathrm{O}_{3}$ VB top.

By combining the PC and the IPE results one can obtain the complete energy band diagram of the Si/oxide interface as sketched in Fig. 3(a). The CB $\left(\Delta E_{C}\right)$ and VB $\left(\Delta E_{V}\right)$ offsets are calculated by subtracting the band gap width of $\mathrm{Si}$ [ $E_{g}(\mathrm{Si})=1.12 \mathrm{eV}$ at $\left.300 \mathrm{~K}\right]$ from the measured barriers $\Phi_{e}$ and $\Phi_{h}$, respectively, and listed in Table I. As the hole IPE barrier $\Phi_{h}$ does not indicate an oxide VB splitting, the lowest portion of the oxide $\mathrm{CB}$ must include $\mathrm{a} \approx 1 \mathrm{eV}$ tail subband as depicted in Fig. 3. The latter is consistent with the observation of a similar tail in the subthreshold PC in the complex oxides. Further reassuring the consistency of the interpretation is that the oxide band gap width derived from the electron and hole IPE barriers $E_{g}(o x)=\Phi_{c}+\Phi_{h}-E_{g}(\mathrm{Si})$ coincides with that directly obtained from the PC analysis. To this must be added that the IPE/PC thresholds are most sensitive to the lowest available band gap/energy barrier at the interface. The average optical band gap width may be even higher than the gap width observed here as suggested by the value $E_{g}$ $=6.2 \mathrm{eV}$ derived from optical measurements for amorphous $\mathrm{LaAlO}_{3}$ (Ref. 15) vs $E_{g}=5.6 \mathrm{eV}$ for $\mathrm{LaAlO}_{3}$ single crystals. ${ }^{2}$

There are two possible explanations for the band tail effect noted above. The first one is applicable to the CB of complex oxides in which the quantum states of the metal cations have significantly different symmetries so that coupling is not allowed. This applies to TM silicate and aluminate alloy systems such as Hf aluminate ${ }^{5,6}$ and titanate, ${ }^{16}$ and $\mathrm{Zr}$ silicate ${ }^{17}$ where the CB states display a two-band behavior. The second explanation involves coupling between TM and RE $d$ states through bonding to the same oxygen. This coupling is illustrated in Fig. 3(b) as applied at stoichiometric compositions such as the $\mathrm{RETMO}_{3}$ oxides of this letter. ${ }^{18}$ In this explanation, the band tail arises because of local symmetry splitting of the threefold degenerate $d^{*}$ states of the RE and TM atoms bonded to the same $\mathrm{O}$ atom. This coupling is observed for the four high-energy states in the $\mathrm{O}$ $K_{1}$ edge of $\mathrm{GdScO}_{3}$ and $\mathrm{DyScO}_{3}{ }^{18}$ Based on a virtual crystal model for $d$-state coupling, it has been proposed ${ }^{5}$ that complex oxides would provide a separate and independent control of the dielectric constant and the CB offset energy. It has been subsequently shown that this model does not apply, and that the localized band-tail states associated with Jahn-Teller effect play the dominant role in determining the band edge electronic structure of the complex oxides of this work.

Though the barrier height for electrons/holes and the dielectric constant are sufficiently high to consider the studied insulators for MOS application, CB tail states represent a potential danger: The effectively reduce the conduction band offset down to $\Phi_{e}^{*}-E_{g}(\mathrm{Si}) \approx 1 \mathrm{eV}$ and enable electron tunneling. The local deviations in the oxide composition and distortions of the RE surrounding may add to a lower energy of the $d^{*}$ states as discussed above. The same trend can be expected if a phase separation in the complex oxide will occur as a result of, for instance, thermal treatment. Therefore, the choice of a particular scandate will be largely determined by its phase separation properties and thermodynamic stability, while the electronic properties are seen to be only weakly sensitive to the sort of RE ion.

To conclude, we have determined the energy band diagram at the interfaces of (100)Si with several RE/TM mixed oxides $\left(\mathrm{LaScO}_{3}, \mathrm{GdScO}_{3}, \mathrm{DyScO}_{3}, \mathrm{LaAlO}_{3}\right)$ and have compared it to that previously determined for elemental $\mathrm{Al}_{2} \mathrm{O}_{3}$ and $\mathrm{HfO}_{2}$. No substantial impact of the RE $4 f$-shell occupancy, varying from 0 in $\mathrm{La}$ to $14 \mathrm{in} \mathrm{Hf}$, on the position of $\mathrm{CB}$ and VB in complex oxides is found. Moreover, the energy of the oxide VB edge appears to be virtually insensitive to the kind of the RE ion. The lowest portion of the oxide $\mathrm{CB}$ is derived mostly from the unoccupied $5 d^{*}$ states of RE ions and this corresponds to a band gap of $\sim 5.6-5.7 \mathrm{eV}$, and a CB offset energy of $\sim 2.0 \mathrm{eV}$. A band tail observed in the IPE measurements is attributed to mixing of RE and TM $d$ states and may have a significant effect on the performance of these complex oxides in gate dielectric applications through a reduction of $\mathrm{CB}$ offset energies to $\sim 1 \mathrm{eV}$.

${ }^{1}$ G. D. Wilk, R. M. Wallace, and J. M. Anthony, J. Appl. Phys. 89, 5243 (2001).

${ }^{2}$ S. G. Lim, S. Kriventsov, T. N. Jackson, J. H. Haeni, D. G. Schlom, A. M. Balbashov, R. Uecker, P. Reiche, J. L. Freeouf, and G. Lucovsky, J. Appl. Phys. 91, 4500 (2002).

${ }^{3}$ M. Leskela and M. Ritala, J. Solid State Chem. 171, 170 (2003).

${ }^{4}$ B. E. Park and H. Ishiwara, Appl. Phys. Lett. 82, 1197 (2003).

${ }^{5}$ G. Lucovsky, Y. Zhang, J. L. Whitten, D. G. Schlom, and J. L. Freeouf, Microelectron. Eng. 72, 288 (2004).

${ }^{6}$ V. V. Afanas'ev, A. Stesmans, and W. Tsai, Appl. Phys. Lett. 82, 245 (2003).

${ }^{7}$ A. V. Prokofiev, A. I. Shelykh, and B. T. Melekh, J. Alloys Compd. 242, 41 (1996).

${ }^{8}$ V. V. Afanas'ev and A. Stesmans, in High- $\kappa$ Gate Dielectrics, edited by M. Houssa (IOP, Bristol, 2004), p. 217.

${ }^{9}$ T. H. DiStefano and D. E. Eastman, Solid State Commun. 9, 2259 (1971).

${ }^{10}$ H. H. Tippins, J. Phys. Chem. Solids 27, 1069 (1966).

${ }^{11}$ H. Öfner, M. G. Ramsey, F. P. Netzer, J. A. D. Matthew, and F. N. Yousif, J. Phys.: Condens. Matter 6, 3453 (1994).

${ }^{12}$ V. V. Afanas'ev, M. Houssa, A. Stesmans, and M. M. Heyns, Appl. Phys. Lett. 78, 3073 (2001).

${ }^{13}$ R. J. Powell, J. Appl. Phys. 41, 2424 (1970).

${ }^{14}$ V. V. Afanas'ev, A. Stesmans, F. Chen, X. Shi, and S. A. Campbell, Appl. Phys. Lett. 81, 1053 (2002).

${ }^{15}$ L. F. Edge, D. G. Schlom, S. A. Chambers, E. Cicerella, J. L. Freeouf, B. Holländer, and J. Schubert, Appl. Phys. Lett. 84, 726 (2004).

${ }^{16}$ V. V. Afanas'ev, A. Stesmans, F. Chen, M. Li, and S. A. Campbell, J. Appl. Phys. 95, 7935 (2004).

${ }^{17}$ G. B. Rayner, D. Kang, Y. Zhang, and G. Lucovsky, J. Vac. Sci. Technol. B 20, 1748 (2002).

${ }^{18}$ G. Lucovsky, Y. Zhang, J. L. Whitten, D. G. Schlom, and J. L. Freeouf, Physica E (Amsterdam) 21, 712 (2004). 